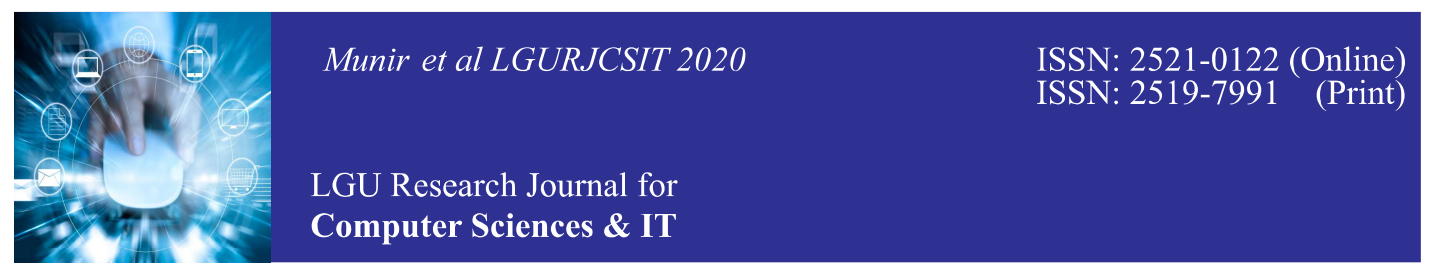

Vol. 4 Issue: 2, April - June 2020

\title{
Cognitive Modelling for User Interface Design in HCI: A Comparative Analysis on Cognitive Models
}

\author{
Sundas Munir ${ }^{1 *}$,Afrozah Nadeem ${ }^{2}$ \\ ${ }^{1}$ Department of Computer Science, Lahore College for Women University, Lahore, Pakistan. \\ ${ }^{2}$ Department of Computer Science, University of Engineering and Technology, Lahore, Pakistan. \\ 1'sundusm1@gmail.com
}

\begin{abstract}
This research aims to elaborate the cognition in the field of human-computer interaction, also acknowledges the cognitive modeling and human behavior processes. Cognitive modeling is a field of Human-Computer Interaction (HCI) which is used to design more efficient human interactive systems. It is used to model the interactive system in such a way that analysts can determine methods that users will interact with the system and also be used to understand the different processes of cognitive human behaviors. Hierarchal task analysis is a task to goal-based model, in which analyst selects the actions and tasks to perform. GOMS is a cognitive knowledge of the human information processing model in HCI that describes the user's cognitive architecture based on four components. The linguistic and grammatical model is a syntactical model in which languages and syntax are designed for the user for system communication in an interactive system. Cognitive human behavior processes are also described to understand the mutual coordination of cognition processes and cognitive models in designing an interactive system. Problem-solving is a cognitive process of the human mind to search for a problem and explore the possible solutions for that problem. Decision making is also a cognitive process of human behaviour in which human chooses an action from other alternatives based on certain criteria.
\end{abstract}

Keywords: Cognition, Cognitive Modeling, Human Behavior, Interactive System, Decision Making, Problem-Solving

\section{INTRODUCTION}

Cognition is the ability to perceive the knowledge from the environment using senses, thoughts, and previous experiences. The infield of HCI, cognition is used to develop the approaches that can envision the usability and interactivity of user interfaces. It helps auser to interact more conveniently with interfaces. It is based on the procedures that how humans interact with technology and how knowledge is transmitted to each other [1]. Cognitive science is a study of mind and its processes such as learning, reasoning, problem-solving and mental representation [2-3]. Mental 
representations are such as attitudes, mental models, schemas, etc. Mental representation is mostly related to behaviours that how a mind behaves towards specific activities and it is also responsible for the change in human behaviours. These mental representations and behaviours are based on mental models and other cognitive processes such as problemsolving, learning and decision making, etc. [4]. Mental models are the sequence of any process and scenario that exists in a human mind. A person uses that mental model to interact with other advanced or new systems. Every person has a different mental model whereas there is a gap between the mental model of a designer and user because the user's mental model can't be so obvious and clear but the designer is an experienced and technical person and his mental model is based on reality and possibilities [5]. Problem-solving is another process of human behaviourthat uses a mental model to find an obvious solution for a problem [6]. Similarly, decision making has been derived from the term problemsolving, it is about how to decide for a problem when there are multiple solutions for a problem but only one solution must be picked for the particular problem[7]. Learning is also a process of cognition in which a user learns the process and usability of the system. So that learnability of the interactive system must be high, the user just sees and he learns the functionality and working of the system [8].

Cognitive Modelling is a field of HCI that is used to understand cognitive processes such as decision making, problem-solving, planning, learning and strategy making, etc. [9]. Cognitive modeling in human-computer interaction isused to develop, promote, and identify the approaches to test the usability of a system before it is built. It is the domain of HCI, which is mostly based on cognitive strategy. A cognitive strategy is a way that helps people to solve their problems by cognitive abilities, so people don't have to remember all the processes and methods, they just have to learn the sequences. Cognitive strategies are created to build a more human-centered system [4]. Also, cognitive strategies are made to predict the performance of tasks in interactive systems [10]. Furthermore, Cognitive processes could not be measured directly so, cognitive models are used to measure the processes indirectly through measuring the human's behaviours by different constraints and sources of cognitive models [9].

\section{Cognitive Modelling in HCI}

Cognitive modelling in HCI is some representation of real systems such as prototypes and other interfaces modules to test and analyse the usability and learnability of the system. Cognitive models are used to predict how a system will respond to the user [11]. Cognitive Modelling has three common uses that are as follows.

i. A cognitive model is used to substitute a human user to analyse how users will use a system.

ii. The system can generate the cognitive models for the user so that it can monitor the interfaces better.

iii. It also would help design and analyse the system for a group 
of people.

It is designed according to user mental models, knowledge, intentions, and understanding. The level of representation varies from technique to technique [12]. Cognitive models are designed to describe a user of interactive systems. There is a need to know how interactive systems work and how their users interact with it [13]. They also enable designers to analyze the system requirements, tasks, and actions that must perform to make interactive systems. Cognitive models are categorized based on their nature that is described below [14]:

1. Hierarchal Task Analysis

2. GOMS Model

3. Linguistic and grammatical models

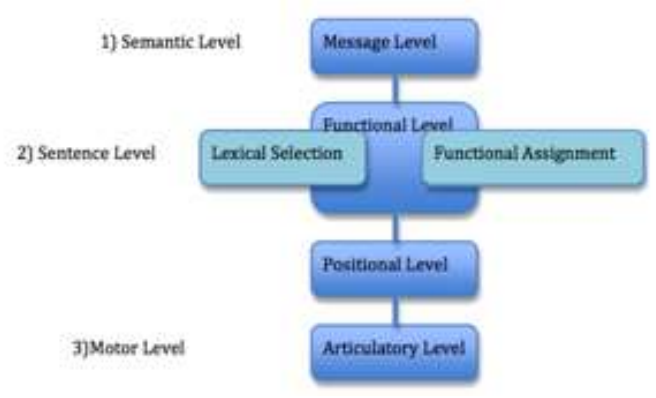

Figure 1: Cognitive modelling levels

\subsection{Hierarchal Task Analysis (HTA)}

The hierarchical model is related to a user's task and goal structures. A hierarchal model can have multiple actions and tasks to achieve a goal. But the designer must have to select a specific task and action. It is a symbolic representation of a task composition that is based on structure charts. Structure charts represent the sequence of tasks and actions as a hierarchy and it also defines whether the actions will be iterative or selective. Selective actions are the structured paths to achieve the goal.

For example, if a user needs to make a call, he can choose two paths. He can search the name of the recipient from the contact list, if the name does not exist then he has to dial a number. Similarly, for visiting a web page, there is a need to follow a structured hierarchy.

In the hierarchal Model, first of all, a task is selected and then an operator is used to apply an operation for that task. When the operation is applied there must be some commands just like moving mouse and push-buttons. After the command layer, there is an action layer where an action is taken to perform a task such as on a keyboard keystroke is a key to go for a certain operation to perform a task to achieve the goal.

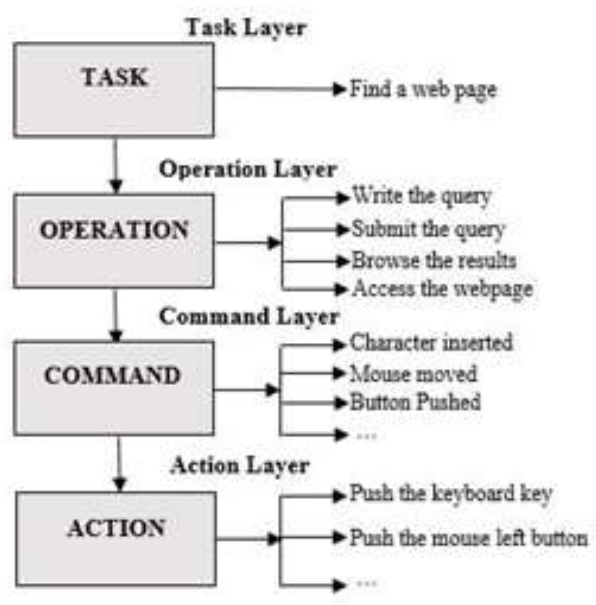

Figure 2: Example for Web page hierarchal model 
This is not a simple model, there must be time spent on describing the hierarchy of tasks and actions. It appears in different methods for interactive systems design. For example, Stanton (2003) uses this model as part of his method for error identification. He develops a method and then uses this model to identify the tasks and actions to detect multiple error situations. At the action level, the user must perform any error when interacting with an interactive system. To prevent this, the analyst must consider all possibilities of error occurrence. Task analysis describes the functionalities of the old system and designer come to know that what would be the needs of a new interactive system.

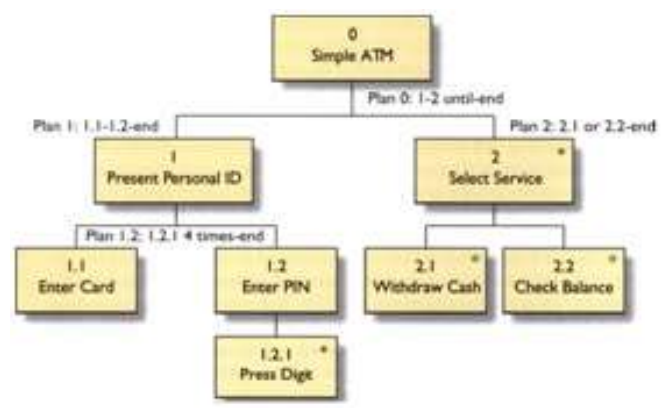

Figure 3: Hierarchical task model for a portion of an ATM

For an ATM machine, hierarchal tasks have to perform to reach the goal. First of all, a person has to select the set of actions. Users can't withdraw money without performing the required tasks. The user must select the personal ID then he would be able to insert the card and enter a pin that would enable him to withdraw the cash. Annett (2004) provides a step-by-step method to perform a hierarchy model:
- Decide the analysis purpose.

- Define the goal of the task.

- Decide what type of data will be collected, how it would be collected, and from where?

- Collect data and make a hierarchical structure diagram.

- Recheck the validity of data.

- Identify all tasks and operations and perform analysis until all possible errors are detected.

- Generate and perform hypothesis tests factors that are affecting learning and performance.

\subsection{GOMS: a family of predictive models}

GOMS is a cognitive knowledge of the human information processing model in HCI that describes the user's cognitive architecture based on four components. The components of GOMS are such as: Goals are symbolic structures that describe what the user wants to achieve and defines the set of possible methods to achieve the goal.

Operators are the actions that the user performs to achieve the goal. The execution of actions is necessary to affect the task environment.

Methods divide the task into sub tasks to describe the procedure for achieving the goal.

Selection Rules are selecting a method or set of specific methods that would be needed when a goal is achieved [17-16]. 


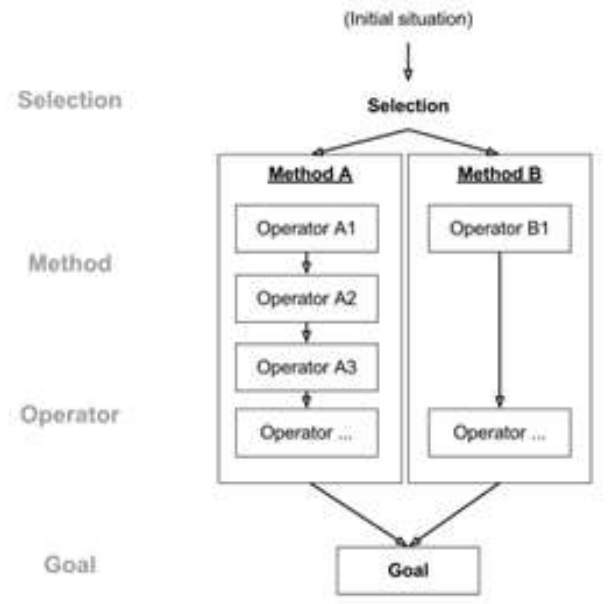

Figure 4: The concept behind a GOMS model and their relationships

GOMS is used to predict how an interactive system will be used by a user. It depends on methods that are composed to achieve goals; these methods are formed up of operators. Operators are the actions that user performs, one or more than one method can be used to achieve a goal, selection rules are used to propose a specific method if one and more than one method is required to achieve a goal [18].

GOMS is a family of Task Analysis methods. 4 basic models of GOMS also called variants of GOMS, which are as follows [19]:

i. Keystroke-Level Model(KLM)

ii. CMN-GOMS

iii. Natural GOMS Language (NGOMSL)

iv. Cognitive, Perceptual, Motors GOMS (CPM-GOMS) [20]

\section{i. Keystroke-Level Model}

The keystroke-level model or KLM is a model that belongs to the family of the GOMS model. This a flexible model that was proposed by Stuart K. Card, Thomas P. Moran, and Allen Newell in their book 'The Psychology of HumanComputer Interaction', that published in 1983. The purpose of this model is to predict how much an expert user takes time to complete a routine task without making any error while using an interactive system [21].It is an iterative model, used for the physical designing of the interactive system. As it is for physical design, so there's no need for enough psychological knowledge. Designers can easily design the system without making a prototype. There are six operators used to develop a system[21-22]:

\section{Physical Motor Operators:}

- $\mathrm{K}$ is a keystroke/button press on the system to interact.

- $\mathrm{P}$ is pointing to an object on a display with a mouse.

- He is directing the hand on the keyboard or another device.

- D is drawing manually.

- Mental Operator:

- $\mathrm{M}$ is a mental operator for mentally preparing to perform actions.

- System Response Operator:

- $\mathrm{R}$ is a response by the system.

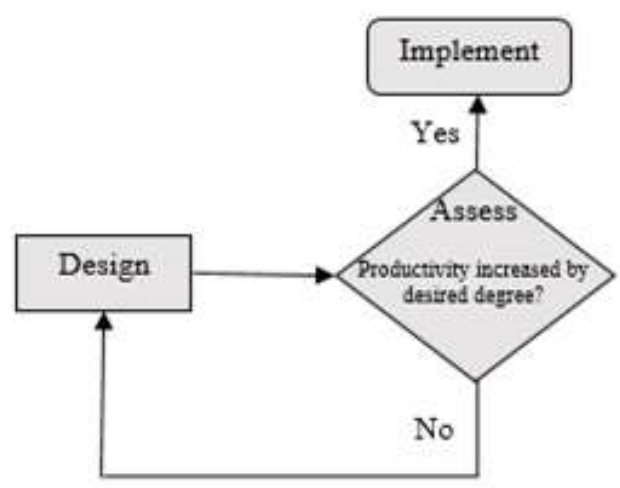

Figure 5: Iterative Process for KLM 
In KLM, the iterative process includes the design of the system and then checks the productivity of the system if yes it enhances the productivity then the design is implemented and if no then again system goes under the design phase.

For Example, there is a task in this example to "delete a file". The operation can be done using two methods:

1. Drag file into the recycle bin.

2. Use the shortcut key to delete the file.

\section{Method A:}

As a task can be done by two methods so the example is divided into two-part in the first part the file is drag into the recycle bin. In the first step user mentally prepare to perform deletion action, in the second step user finds the icon which he wants to delete. In the third step user point the icon using the mouse on the screen. The fourth user presses the mouse button and holds the button for drag operation. In the fifth step file icon is dragged to over the recycle bin icon and then the mouse button is released in the sixth step. In the last step user point to the original window. In this keystroke test three pointing, two keystrokes, and two mental prepare operators are used, so this task performed in 6.2 seconds.

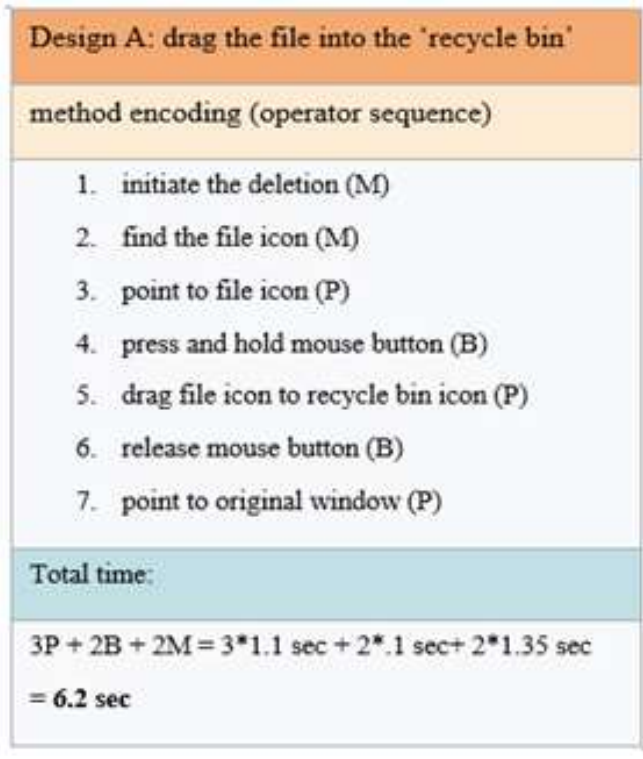

Figure 6: Design A drag a file into recycle bin

\section{Method B:}

In the second method, deletion operation is performed by using the shortcut key 'Del/Delete' form keyboard. In this method user first, mentally prepare himself to perform deletion operation, then in the second step find the file icon that the user wants to delete. In the third step point the mouse icon and in fourth and fifth step the user press and release the mouse button. In the sixth step, the user moves his hand from the mouse to the keyboard and presses the Del/Delete button. In the last step, the user moves his hand back to the mouse. In this method one pointing, three keystrokes, two homing hands, and two mental prepare. Operators are used and an expert user takes 5 seconds to perform this task. This results that method B is more time effective as compared to $\operatorname{methodA}[21]$. 


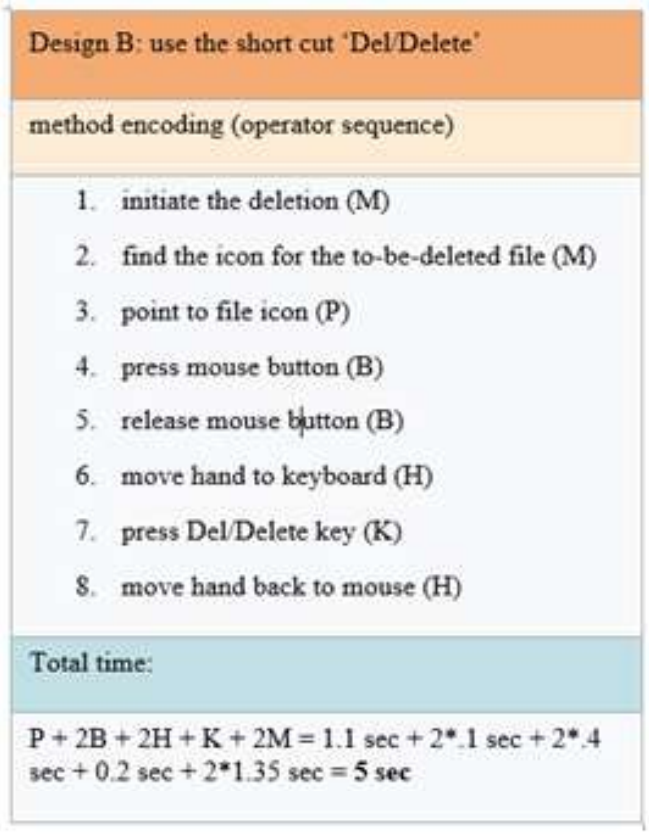

Figure 7: Design B use shortcut "Del/Delete"

\section{ii. CMN-GOMS}

CMNS-GOMS is an original technique of GOMS. The abbreviation of CMNGOMS is Card, Moran, and Newell GOMS[23].

In this technique, the GOMS four components "goal, operation, method, and selection rule" is more strictly followed. Its structure is also more rigid as compared to GOMS other types, it means the evaluated tasks using this technique are always in the format of pseudo-code and no other formal syntax is allowed. It also provides a complete guide to formulate the selection rules for a particular problem. The advantage of this technique is that it eliminates the load of the task that is placed on the user [24].

There is a simple example of CMNGOMS in which user shave to take an image using the iPhone. As it is discussed above that in this technique the tasks are evaluated in the form of pseudo-code, so this example is described as pseudo-code format. The main goal of the user is to take a picture from the iPhone, so users divide the main goal into three sub-goals. In the first goal, the user reaches the iPhone, opens it, tap on the camera application icon then wait for a while to open the application, and at the end of the first goal, the user verifies that application is the start or not.

\section{GOAL: TAKE PICTURE ON IPHONE}

GOAL: START-APPLICATION

- REACH -FOR-IPHONE

- RETRIEVE-HOME-SCREEN

- tap-Application-icon

- WATT-FOR-APPLICATION-LOAD

- VERIfy-APPLication-LOAD

GOAL: CAPTURE-SUBJECT-PHOTOGRAPH

- CHANGE-GAZE-TO-SUBJECT

- POINT-CAMERA-TOWARD-SUBJECT

- VERIFY-FACE-DETECTED

- tap-Shutter-LCD-BUtTon

- VERIFY-APPLICATION-LOADING

GOAL: VERIFY-APPLICATION-RESPONSE

- WATT-FOR-APPLICATION-RESPONSE

- VERIFY-IMAGE-RESPONSE

Figure 8: CMN-GOMS

Take a picture on iPhone

In the second goal, the user focus on the subject points the camera angle to the subject to take its photo, detects the subject face or body, clicks on the shutter button to take an image, and in the last user checks the application loading. In the third and last goal, users wait for application response and see the image. The task is complete. 


\subsection{Linguistic and grammatical models}

Linguistic and grammatical models are the models which are used to communicate between user and systems. As a cognitive model, this is the model of symbols and syntactic grammar that would be helpful in the perception of the concept and understanding the purpose of the system. Such as in interactive interface designing, the designer uses some icons to represent a software as well as he uses a specific keyword. For example, Microsoft Office is a software which has a specific icon, the user just sees the icon and recognize the software but as well as he also reads software name to confirm that it is the required software. Further when the user starts using software their perceptual symbols and keywords just like file, save, open these words are specifically used for obvious purposes so, the user just looks at the keywords and learns the software by his previous knowledge and gets familiar in meantime.

This model has some of the techniques such as:

- Backus-Naur Form (BNF)

- Task-Action Grammar (TAG)

\section{Backus-Naur Form (BNF)}

BNF technique isused to define the syntax of a language such as document formats, computer programming languages, instruction sets, and communication protocols. It describes context-free grammars where the exact meaning of a language is required. BNF describes a language in terms of
Terminal symbols, production rules, and syntactic constructs. BNF uses a set of derivation rules and it is written as:

$<$ symbol $>::=$ _expression

In this above equation, <symbol $>$ is a non-terminal and expression consist more sequence of symbols, Terminal symbols are the symbols of a language, such as punctuation and words. Syntactic constructs are phrases, statements, and sentences, etc. Some symbols use for grammar[25].

$::=$ means 'is defined as'

is 'or'

$<>$ is to write 'category names'

Let us see an example of BNF for numbers:

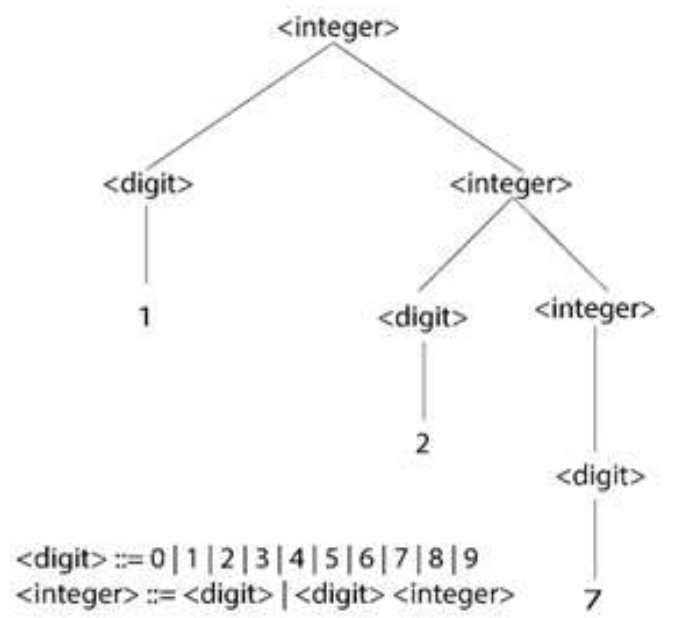

Figure 8: BNF for grammar related to numbers

There is grammar for digits in which digit and integers are non-terminal and numbers are terminals. $<$ digit $>$ can be any number between $0-9$. <integer $>$ non-terminal defines the digits; an integer could be one digit or digit that is followed by an integer that could be further one digit or digit followed by 
another integer and so on. This grammar removes the need to list all possible integer combinations of digits [26].

There are variants for Backus Naur Form which are:

- Extended Backus-Naur form (EBNF)

- Augmented Backus-Naur form (ABNF)

\section{A C O M P A R A T I V E ANALYSIS OF COGNITIVE MODELS}

Cognitive models are designed to improve the usability and learnability of systems to make interactive systems more human-centered.Through cognitive modeling, designers can make such new systems that have more interactivity and usability than the old system.

Hierarchal Task Analysis is a sequential method that selectively performs tasks and actions, it decides the goal and then performs tasks accordingly. User shave to perform all actions that are needed to achieve a goal. It is easy to use and learn also it can be applied to different types of physical and mental activities to improve the analysis. Hierarchal task analysis needs a proper team to work with it because it takes so much effort and time to use. It is not for parallel activities and only works for procedural activities. Errors in hierarchal task analysis are inevitable in the performance of the task [27].

GOMS models are predictive modelsso they give qualitative and quantitative measures for the actions and tasks. User
Interfaces can be easily modifiable. GOMS is simple, cost-effective, and less time-consuming in calculating the interaction estimation [28]. For this purpose, the data for analysis must be accurate. GOMS produced the most valid and accurate predictions [29]. As GOMS modelsare mostly based on the prediction of user methods and take a lot of effort, skills, and time but they still not be able to remove the unpredictability of users because they do not consider the user behaviours[29]. It only applicable for goal-directed tasks and needs an expert to perform tasks because it does not support errors[30].

Linguistics and grammatical models are used for syntactical grammar that would be helpful in user to system interaction. They are based on contextfree grammar. BNF is used to define the syntax of a language like computer programming languages, document formats, instruction sets and communication protocols, etc. It simplifies the programming languages so that they become easier to understand. It is very complex to deal with this technique because there is the same syntax used for different semantics. There is also no knowledge of human/user perception of knowing whether the user knows the semantics or not and also there is minimum consistency checking because of reuse of the same syntax [16].

KLM is used for the physical designing of the interactive system based on actual knowledge of the human motor skill system that depends upon the maturation of the central nervous system of humans. It is derived from GOMS, the lowest level of it but KLM 
is comparatively easy to use because it's a physical design. Users are different regarding their motor skills, technical ability, knowledge, and tasks[17,21].

Hierarchal task analysis and GOMS models are nearly similar that they both examining the goals and sub-goal analysis but there are some differences that GOMS depends on user predictions but HTA does not need user predictions. HTA is easy to use and learn but GOMS is comparatively complex and needs experts for analysis.

Hierarchal task analysis and Linguistics models are different because hierarchal models are semantic level models whereas linguistic models are syntactical level models. BNF and GOMS are also different, such as GOMS is predictive goal-directed models but BNF is selective models. There is no human perception in BNF but GOMS models use the user prediction methods. GOMS has consistent goals and tasks but there is minimum consistency in BNF. The same syntax could be used many times for different sentences.

KLM is mostly similar to HTA and GOMS because it is a derived model of GOMS. It also has operators and methods to perform analysis. Similar to HTA it is a step by step method. Like GOMS and HTA it also doesn't support errors, so that method execution must be error-free and like GOMS only an expert can deal with it. KLM is a physical and device-level model and includes motor skill operators.

\section{CONCLUSION}

This article concludes that cognition and cognitive modeling is very important in the field of HCI. Cognitive models help designers and analysts to make more efficient interactive systems that would be improved forms of current systems. Analysts use these models to detect errors and deficiencies in their systems. They also eliminate errors by using these models. In this research, categories of cognitive models have been discussed and their comparative study has been performed. There is a hierarchal task analysis that is the task to goal method in which a goal is set, and a sequence of actions has been performed to achieve that goal. GOMS models are the family of the task analysis model which is used to predict how an interactive system will be used by a user. It depends on methods that are composed to achieve goals; these methods are formed up of operators. Operators are the actions that user performs, one or more than one method can be used to achieve a goal, selection rules are used to propose a specific method if one and more than one method is required to achieve a goal. There are further methods of GOMS but in this research, two methods have been elaborated. KLM keystroke Level model is a model that belongs to the family of the GOMS model. The purpose of this model is to predict how much an expert user takes time to complete a routine task without making any error while using an interactive system [21]. It is an iterative model, used for the physical designing of the interactive system. As it is for physical design, so there's no need for enough psychological knowledge. Designers can easily design the system without making a prototype. In CMN-GOMS, the GOMS's four components "goal, operation, method, and selection rule" 
are more strictly followed. Its structure is also more rigid as compared to GOMS other types, it means the evaluated tasks using this technique are always in the format of pseudo-code and no other formal syntax is allowed. Similarly, BNF is also discussed in this article which is used to define the syntax of a language like a computer programming languages, document formats, instruction sets and communication protocols, etc. It simplifies the programming languages so that they become easier to understand. It is very complex to deal with this technique because there is the same syntax used for different semantics. There is also no knowledge of human/user perception of knowing whether the user knows the semantics or not and also there is minimum consistency checking because of reuse of the same syntax. HTA, GOMS, and Linguistic Models are used for similar purposes to make user-centered and human interactive systems but they are slightly different in some aspects. HTA is semantic analysis method, linguistics models are syntactic level models whereas GOMS is mostly similar to HTA but there are some differences that GOMS depends on user predictions but HTA does not need user predictions. HTA is easy to use and learn but GOMS is comparatively complex and needs experts for analysis. So, these models make designs and analysis better and easy. Human processes are also involved in these analyses to make better systems.

\section{References}

[1] J. Hurtienne, "COGNITION IN HCI: AN ONGOING STORY," Human Technology: An Interdisciplinary
Journal on Humans in ICT Environments, p. 17.

[2] "Cognitive science," Wikipedia, the free encyclopedia, [ O n 1 i n e ]. A v a i 1 a b 1 e : https://en.wikipedia.org/wiki/Cognitiv e_science.

[3] R. L. Boring, "HUMANCOMPUTER INTERACTION AS COGNITIVE SCIENCE," p. 5, 2015.

[4] "Cognitive Capabilities: Mental Representations, Problem Solving and Decision Making".

[5] J. K. Doyle, "The cognitive psychology of systems thinking," RESEARCH PROBLEMS.

[6] V. C. Yingxu Wang *, "On the cognitive process of human problem solving," Science Direct, p. 12, 2008.

[7] G. R. Yingxu Wang, "The Cognitive Process of Decision Making," Int'l Journal of Cognitive Informatics and Natural Intelligence, p. 13, 2007.

[8] A. B. S. W. a. N. R. Sabine Prezenski1*, "A Cognitive Modeling Approach to Strategy Formation in Dynamic Decision Making," Original Research ARTICLE, 2017.

[9] J. A. W. a. D. R. Murugesh, "Cognitive Modeling in Human Computer Interaction for Aging: Prior Experience and Perception of Technology - A Pilot Study," Int'l J o u r a l of Computing, Communications \& Instrumentation Engg, vol. 4, no. 1, p. 6, 2017. 
[10] A. Hornof, "The Role of Cognitive Strategy in HumanComputer Interaction," The HumanComputer Interaction Institute, [ O n $1 \mathrm{ine}$ ]. Ava i 1 a b 1 e : https:/hcii.cmu.edu/news/seminar/eve nt/2016/01/role-cognitive-strategyhuman-computer-interaction.

[11] Margaret-Rouse, "cognitive modeling," TechTarget, [Online].

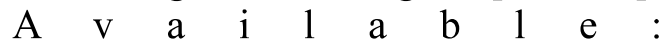
https://searchenterpriseai.techtarget.co $\mathrm{m} /$ definition/cognitive-modeling.

[12] B. G. John, "Cognitive Modeling for Human-Computer Interaction," Graphics Interface '98, p. 7.

[13] W. D. G. \&. E. M. Altmann, "COGNITIVE MODELING AND H U M A N - C O M P U T E R INTERACTION," Research Gate, p. 22, 1999.

[14] "Cognitive Framework:Modes of Cognition".

[15] D. Benyon, Designing Interactive Systems A comprehensive guide to $\mathrm{HCI}, \mathrm{UX}$ and interaction design, United Kingdom: PEARSON, 2014.

[16] "cognitive models," [Online].

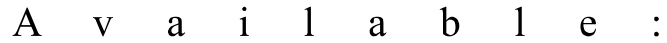
https://hcibook.com/e3/chaps/ch12/out line/.

[17] "GOMS," Wikipedia, the free encyclopedia, [Online]. Available: https://en.wikipedia.org/wiki/GOMS.

[18] J. R. O. a. G. M. Olson, "The Growth of Cognitive Modeling in
Human-Computer Interaction Since GOMS".

[19] "Task Analysis," [Online].

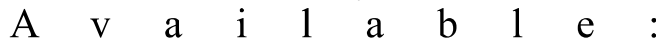
https://web.cs.dal.ca/ jamie/teach/Nic kGibbins/task-analysis.html.

[20] "GOMS," Usability Body of Knowledge, [Online].Available: https://www.usabilitybok.org/goms.

[21] "Keystroke-level model," [ O n line]. Ava i 1 ab le : https://en.wikipedia.org/wiki/Keystrok e-level_model\#Advantages.

[22] O. G. \&. F. Ritter, "Keystroke Level model".

[23] "GOMS: Quick to calculate, Tough to make sense," cs3240 Team 13, [ O n 1 i n e]. Av a i 1 a b 1 e : https://cs3240team13.wordpress.com/ 2012/11/02/goms-quick-to-calculatetough-to-make-sense/.

[24] "CMN-GOMS," Wikipedia, [ O n 1 i n e]. A va i 1 a b 1 e : https://en.wikipedia.org/wiki/CMNGOMS.

[25] "Backus-Naur form," Wikipedia, the free encyclopedia, [ O n 1 i n e ]. A v a i l a b le : https://en.wikipedia.org/wiki/Backus\% E2\%80\%93Naur_form.

[26] "B ackus-Naur Form," MultiWingSpan, [Online]. Available: http://www.multiwingspan.co.uk/a23. php?page $=$ bnf.

[27] "Ergonomics Methods and Tools," [Online]. Available: http://www.ul.ie/ infopolis/methods/in 
dex.html.

[28] "Advantages of The GOMS Model," TRIPOD, [Online]. Available: http://members.tripod.com/elena_chmi 1/thegomsmodel/id4.html.

[29] "GOMS," CS3240 Team 11, [O n l i n e ]. A va i l a b le : https://cs3240team11.wordpress.com/ 2012/10/26/goms/.

[30] "State the disadvantages of GOMS(Goals, Operators, Methods, and Selection rules)," Kenyaplex.com, [ O n line ]. A v a i l a b l e : https://www.kenyaplex.com/questions/ 29863 -state-the-disadvantages-ofgoms-goals-operators-methods-andselection-rules.aspx. 